\title{
Refinement of high-risk endometrial cancer classification using DNA damage response biomarkers: a TransPORTEC initiative
}

\author{
Aurélie Auguste $\mathbb{1}^{1} \cdot$ Catherine Genestie $\mathrm{C}^{1,2} \cdot$ Marco De Bruyn $^{3} \cdot$ Julien Adam ${ }^{2} \cdot$ Audrey Le Formal $^{1}$. \\ Françoise Drusch ${ }^{4} \cdot$ Patricia Pautier ${ }^{5} \cdot$ Emma J. Crosbie $^{6}{ }^{6} \cdot$ Helen MacKay ${ }^{7} \cdot$ Henry C. Kitchener ${ }^{6} \cdot$ Melanie Powell $^{8}$. \\ Pamela M. Pollock ${ }^{9} \cdot$ Linda Mileshkin $^{10} \cdot$ Richard J. Edmondson $^{10,11} \cdot$ Remi Nout $^{12} \cdot$ Hans W. Nijman $^{13}$. \\ Carien L. Creutzberg ${ }^{12} \cdot$ Tjalling Bosse $^{14} \cdot$ Alexandra Leary $^{1,5}$
}

Received: 1 August 2017 / Revised: 30 March 2018 / Accepted: 30 March 2018 / Published online: 28 June 2018

(c) United States \& Canadian Academy of Pathology 2018

\begin{abstract}
The TransPORTEC consortium previouslclassified high-risk endometrial cancer including poor-risk histologies such as clear cells, into four molecular subtypes "POLE mutated," "microsatellite unstable," "TP53 mutated," and "no specific molecular profile." We evaluated whether DNA damage response biomarkers could further refine this high-risk tumors classification, in particular the heterogeneous "no specific molecular profile" and "TP53 mutated" subsets recently qualified as poor prognosis in high-risk endometrial cancer. DNA damage response biomarkers including proteins involved in DNA damage ( $\delta$-H2AX), homologous recombination (RAD51), regulators of error-prone Non Homologous End-Joining (DNA-pk, FANCD2), and PARP-1 were evaluated in 116 high-risk tumors by immunohistochemistry. CD8 and PD-1 expression by immunochemistry and mutation analyses were performed previously. Survival outcome were calculated using Kaplan-Meier and Log-rank test. None of the DNA damage response biomarkers alone were prognostic. However markers were informative within molecular subsets. Among the "no specific molecular profile" subset, $\delta-\mathrm{H} 2 \mathrm{AX}+$ was significantly predictive of poor disease free survival (Hazard Ratio $=2.56 ; p=0.026$ ), and among "TP53 mutated," a DNA-pk+/FANCD2- profile (favouring errorprone Non Homologous End-Joining) predicted worst disease free survival (Hazard Ratio $=4.95 ; p=0.009$ ) resulting in five distinct prognostic subgroups from best to worst prognosis: group1 "POLE mutated/Microsatellite unstable" $>$ group2 "no specific molecular profile with no DNA damage" > group3 "TP53 mutated/Non Homologous End-Joining negative"> group4 "no specific molecular profile with high DNA damage" > group5 "TP53 mutated/Non Homologous End-Joining positive"; $p=0.0002$ ). Actionable targets were also different among subsets. Group3 had significantly higher infiltration of PD-1+ immune cells $(p=0.003)$, segregating with group1. Group2 had frequent PI3K pathway mutations and ER positivity. While group5, with the worst prognosis, had high DNA damage and PARP-1 expression providing a rationale for PARP inhibition. Our findings have refined the TransPORTEC prognostic classification of high-risk endometrial cancer into five distinct subgroups by integrating DNA damage response biomarkers and identified molecular subtype-specific therapeutic strategies.
\end{abstract}

Electronic supplementary material The online version of this article (https://doi.org/10.1038/s41379-018-0055-1) contains supplementary material, which is available to authorized users.

Aurélie Auguste

aurelie.auguste@gustaverousy.fr

$\triangle$ Alexandra Leary

alexandra.leary@gustaveroussy.fr

Extended author information available on the last page of the article

\section{Introduction}

The majority of epithelial endometrial cancers have an excellent prognosis and will likely be cured after local treatment alone [1]. Most of these tumors are low grade endometrioid tumors, frequently express hormone receptors and tend to present at a localized stage-their 5 year overall survival is excellent at $85-90 \%$. However, a subset of endometrial cancers $(15 \%)$ has a poor prognosis. Clinicopathological factors have been proposed to identify the subgroup of patients at increased risk of relapse after local treatment alone [2]. These include grade three endometrioid 
or non-endometrioid histology (high grade serous or clear cell), lymphovascular invasion and advanced stage (II or III). Although it is well established that a proportion of endometrial cancers are at high-risk of relapse, there are few data to support the use of adjuvant chemotherapy. In fact the recently presented PORTEC- 3 trial evaluating the benefit of adjuvant platinum-based chemotherapy in high-risk endometrial cancer failed to show a significant increase in overall or failure free survival [3]. In univariate analyses the only parameter associated with chemotherapy benefit was stage III disease. There is clearly a need to improve the genomic and molecular characterization of high risk endometrial cancer beyond currently available clinicopathological factors.

Recent studies have provided valuable insight into the genetic heterogeneity of endometrial cancers and helped refine their prognostic classification. The TCGA performed comprehensive genomic profiling of over 300 endometrial tumors resulting in a new genomic prognostic classification for endometrial cancers: POLE mutated endometrioid tumors, microsatellite unstable tumors, microsatellite stable copy number-low and copy number-high serous-like endometrial cancers (mainly serous histology associated with TP53 mutations) [4].

We and others have validated more cost-effective and clinically applicable methods for molecular classification of Endometrial Cancer using targeted sequencing and immunohistochemistry on formalin-fixed paraffin-embedded tumor samples [5, 6]. In addition, the TCGA analyses were conducted on mainly low-risk endometrial tumors. However refined prognostication of endometrial cancers is most urgently needed for its high-risk subset where this information could guide adjuvant treatment decisions and identify novel targeted strategies. The TransPORTEC Consortium was established for translational research in high-risk endometrial cancer and has previously validated a simple molecular classification resulting in four distinct prognostic subgroups "POLE mutated," "microsatellite unstable," "TP53 mutated" (as a surrogate for copy numberhigh) and "no specific molecular profile" in high-risk endometrial cancer where tumors were classified as highrisk according to the definition used in the PORTEC3 trial (Trial register http://www.trialregister.nl/trialreg/admin/ rctview.asp?TC $=729$ 2014). It is noteworthy that Progression Free Survival curves almost overlap for "TP53 mutated" and "no specific molecular profile" which could be explained by high number of non endometrioid, such as clear cells Endometrial Cancer.

In this high-risk endometrial cancer cohort, we confirmed that the "POLE mutated" and "microsatellite unstable" molecular subsets had a favorable prognosis and demonstrated significantly higher immune infiltration and PD-1 and PD-L1 expression providing the rationale for immune targeted strategies in these two molecular subgroups of endometrial cancers [7].

Studies investigating DNA repair competency in endometrial cancers have largely focused on the "POLE mutated" and "microsatellite unstable" subsets which demonstrate defects in the mismatch DNA repair pathway resulting in high mutational burden. However much less is known about the other genomic subgroups. In particular, the copy number-high pattern detected in a significant proportion of endometrial cancer may suggest defective DNA repair capacity secondary to impaired homologous recombination. Data suggest that contrary to High Grade Serous Ovarian Cancer or triple negative breast cancer, high grade endometrial cancers rarely demonstrate BRCA mutations that could account for this genomic instability. Given the plasticity and redundancy of DNA repair pathways, other DNA repair pathways may be relevant. For example, tumor cells favoring "error-prone" Non-Homologous End-Joining over Homologous Recombination may also show similar DNA damage repair defects, and as such measures of Non Homologous End-Joining may offer prognostic information. An evaluation of the DNA damage response of individual tumors may provide valuable insight into prognosis and sensitivity to adjuvant platinum-based chemotherapy.

The first aim of the current study was to investigate whether DNA damage response biomarkers could further refine the prognostic classification of high risk endometrial cancer, in particular the "no specific molecular profile" and "TP53 mutated" molecular subsets as they were characterized as poor prognosis subgroups in high-risk endometrial tumors subpopulation. The second aim was to further characterize the "no specific molecular profile" and "TP53 mutated" subsets in order to inform the selection of patients for adjuvant chemotherapy, immune therapies, DNA damage response inhibitors, or other targeted agents.

DNA damage response biomarkers involved in DNA damage $(\delta-\mathrm{H} 2 \mathrm{AX})$ and response (homologous recombination (RAD51), positive or negative regulators of error-prone Non Homologous End-Joining (DNA-pk or FANCD2, respectively), and PARP-1 were evaluated in a cohort of 116 high-risk endometrial cancers (Fig. 1) and correlated to disease-free survival as well as to actionable alterations and immune profiles.

\section{Materials and methods}

\section{Patient and tissue collection}

The high risk endometrial cancer cohort was composed of 116 tumors (including endometrioid, serous, and clear cells histotypes) as previously described [6] collected from TransPORTEC consortium partner institutions. High-risk 
Fig. 1 DNA damage response biomarkers (in red). SSB Single Strand Break, DSB Double Strand Break, NHEJ Non Homologous End-joining, HR Homologous Recombination, $B E R$ Base Excision Repair, NER Nucleotide Excision Repair, MMR Mismatch Repair

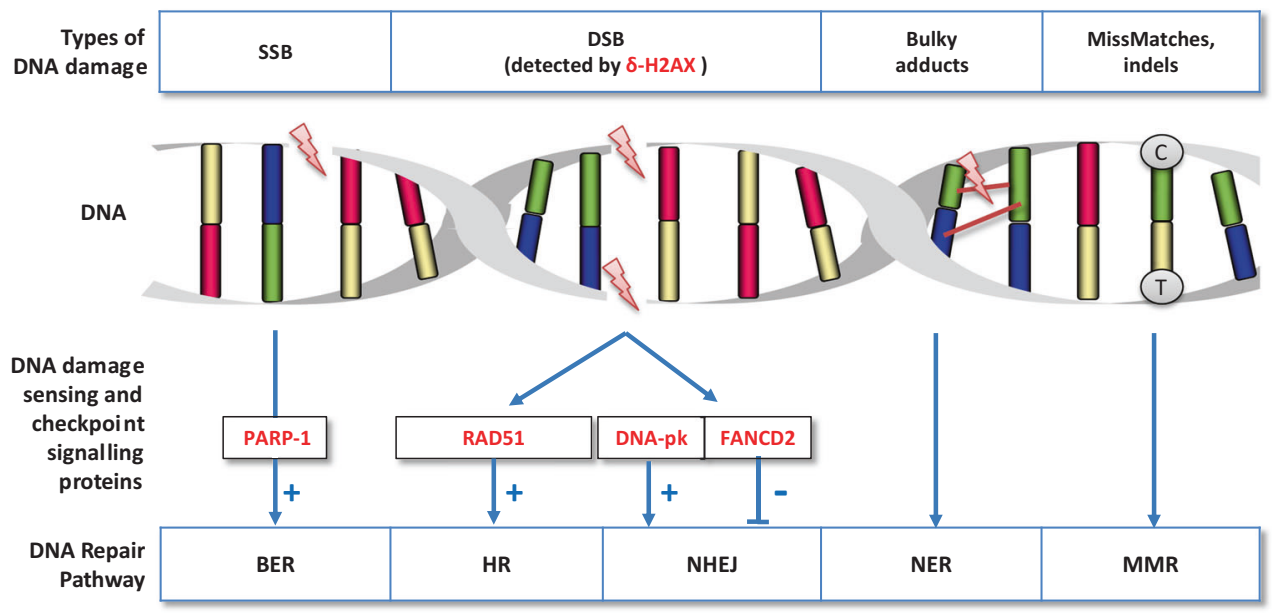

criteria were as per PORTEC3 protocol. Post-operative treatment included either radiotherapy $(N=66)$, chemotherapy $(N=5)$, both $(N=11)$, or no adjuvant therapy $(N=10)$. For 24 patients treatment details were unknown.

\section{Tissue microarray and immunohistochemistry}

TMAs were constructed as previously described using triplicate $1 \mathrm{~mm}$ cores from tumor regions selected by two specialist gynecological pathologists (VS and TB) with $>70 \%$ tumor cellularity. Immunostaining was performed on Tissue Microarray sections using DNA repair biomarkers (DNA-pk, FANCD2, RAD51, and PARP-1) as well as DNA damage biomarker, $\delta$-H2AX as per protocols described in Table S1.

\section{Immunohistochemistry scoring and analysis}

Biomarker scoring was performed in a blind way by two independent anatomo-pathologists (CG and JA) using the $\mathrm{H}$-score method (range $0-300$ ) which is the product of intensity (1-3) and percentage of positive cells (1-100) (see additional information on supplemental methodology). Tumors were scored negative if $\mathrm{H}-\mathrm{Score}<10$. Only tumors with available data for the five DNA damage/repair biomarkers and clinical data were included for the refined prognostic classification $(N=102 / 116)$ (Table $\mathrm{S} 2)$. The aim of analyzing protein expression of DNA damage response biomarkers by immunochemistry was to evaluate the prevalence and prognostic significance of complete absence of expression of each DNA damage response biomarker (Figure S1). Therefore the cut-off was an H-score threshold of $\leq 10$ out of a total possible 300 to define biomarkernegative tumors, the hypothesis being that only the complete lack of detectable DNA repair effector would be biologically meaningful. Correlation between different markers in individual tumors was low (for example for $\delta$ H2AX spearman $r$ range: $0.002-0.286$ when compare to RAD51, DNA-pk, PARP, and FANCD2) suggesting that tissue hypoxia, time to fixation, or other pre-analytical conditions did not affect marker expression (See Table S3). The three cores from each tumor were randomly distributed throughout the Tissue microarray and scores obtained for the three cores were remarkably consistent (e.g., for DNApk (spot1\&2): $r=0.80, p=10^{-25} ; \delta-\mathrm{H} 2 \mathrm{AX}(\operatorname{spot} 1 \& 2): r=$ $0.84, p=10^{-29}$ ) demonstrating both reproducibility of scoring and low level intratumoral heterogeneity for the chosen biomarkers.

\section{Other biomarker analyses}

TP53, POLE mutations, and microsatellite instability status were evaluated as previously described [6]. In the case of concomitant molecular alterations tumors were classified as "POLE mutated" if a mutation was detected, regardless of microsatellite instability or TP53 mutation and then as "Microsatellite unstable" regardless of TP53 status. In addition, results from targeted next generation sequencing was available for 159 hotspot mutations in 13 genes (BRAF, CDKNA2, CTNNB1, FBXW7, FGFR2, FGFR3, FOXL2, HRAS, KRAS, NRAS, PIK3CA, PPP2RIA, and PTEN) as previously published in Stelloo et al. [6].

\section{Statistics analysis}

Differences in medians between two groups were assessed using Mann-Whitney and using ANOVA for three groups or more. Overall survival and disease free survival were calculated using the Kaplan-Meier method and Log-rank test starting at the date of diagnosis. For Disease Free Survival analysis, all recurrences irrespective of type (local, regional, and distal) and death were considered as an event. For Overall Survival, all deaths irrespective of cause were 
considered as an event. Differences between survival curves were evaluated using Mantel-Cox. Graph Pad Prism 5.0 software was used for all statistical analyses. Maximal follow-up was capped at 120 months.

\section{Results}

\section{DNA damage in high risk endometrial cancer}

The H-score for each biomarker was assessed in the whole cohort $(N=116$; see supplemental Figure S2) and analysis performed on tumors with available data on all DNA damage response biomarkers $(N=102)$ (Table S2). Highrisk endometrial cancers were categorized according to the previously published TransPORTEC refined classification for High-risk endometrial as "Microsatellite unstable/POLE mutated" $(N=28)$, "TP53 mutated" $(N=37)$, and "no specific molecular profile" $(N=37)$ in the cohort with data available on all biomarkers. Among the cohort of 102 patients with data available on all biomarkers, POLE mutation and Microsatellite instability remained predictive of improved Disease Free Survival (Hazard Ratio $=2.60 ; p$ $=0.007$ and Hazard Ratio $=2.75 ; p=0.04$, respectively), while TP53 mutations were markers of poor prognosis (Hazard Ratio $=2.06 ; p=0.03$ ) (Table 1). Among highrisk endometrial cancer tumors evaluable for all markers, 69\% (70/102) demonstrated detectable 8 -H2AX (Fig. 2), a measure of double strand break DNA damage. GammaH2AX did not correlate with grade, histology, or patient age in this cohort, however median $\delta$-H2AX levels were significantly higher in "TP53 mutated" high-risk endometrial cancer than in the "Microsatellite unstable/POLE mutated" $(p=0.03)$ or "no specific molecular profile" $(p<0.0001$, Figure S3).

\section{DNA repair biomarkers in high-risk endometrial cancer}

A high proportion of high-risk endometrial cancer showed complete loss of DNA repair proteins with $27 \%(28 / 102)$ negative for RAD51, 43\% (44/102) for DNA-pk and 44\% (45/102) for FANCD2 (Table S2, Fig. 2 \& S2). In contrast most (95\%) tumors retained detectable levels of PARP-1 and median PARP-1 H-score was 175 (Figure S2). Not surprisingly, $\delta$-H2AX and RAD51 levels were positively correlated $(R=0.318 ; p=0.001)$, however, among RAD51 null tumors 57\% (16/28) demonstrated DNA damage. Neither $\delta-\mathrm{H} 2 \mathrm{AX}$ nor individual DNA repair proteins were prognostic for Disease Free Survival or Overall Survival, although loss of RAD51 expression (candidate biomarker for Homologous Recombination deficiency) was associated with a trend
Table 1 Biomarker status and survival outcome in high-risk endometrial cancer $(N=102)$

\begin{tabular}{|c|c|c|c|c|c|}
\hline Pathways & Marker & $\begin{array}{l}\text { Number } \\
\text { of tumors }\end{array}$ & $\begin{array}{l}\text { Median } \\
\text { survival } \\
\text { (months) }\end{array}$ & $\begin{array}{l}\text { Hazard } \\
\text { Ratio for } \\
\text { DFS }\end{array}$ & $p$-Value \\
\hline \multirow[t]{6}{*}{ MMR } & POLE & & & & $*$ \\
\hline & + & 9 & unreached & 2.600 & 0.0379 \\
\hline & - & 93 & 45.4 & & \\
\hline & MSI & & & & $* *$ \\
\hline & + & 21 & unreached & 2.755 & 0.0067 \\
\hline & - & 79 & 39.7 & & \\
\hline \multirow[t]{3}{*}{ Cell cycle } & TP53m & & & & $*$ \\
\hline & + & 38 & 36.5 & 2.056 & 0.028 \\
\hline & - & 64 & unreached & & \\
\hline \multirow{3}{*}{$\begin{array}{l}\text { DSB DNA } \\
\text { damage }\end{array}$} & $\delta-\mathrm{H} 2 \mathrm{AX}$ & & & & \\
\hline & + & 70 & 39.7 & 1.786 & 0.0829 \\
\hline & - & 32 & unreached & & \\
\hline \multirow[t]{3}{*}{ HR } & RAD51 & & & & \\
\hline & + & 74 & 45.0 & 1.489 & 0.2448 \\
\hline & - & 28 & unreached & & \\
\hline \multirow[t]{6}{*}{ NHEJ } & DNA-pk & & & & \\
\hline & + & 58 & 22.6 & 1.541 & 0.1043 \\
\hline & - & 44 & 52.0 & & \\
\hline & FANCD2 & & & & \\
\hline & + & 57 & 52.0 & 1.116 & 0.7274 \\
\hline & - & 45 & 45.4 & & \\
\hline \multirow[t]{3}{*}{ NER } & PARP-1 & & & & \\
\hline & + & 97 & 45.4 & 2.983 & 0.0659 \\
\hline & - & 5 & unreached & & \\
\hline
\end{tabular}

P53 and POLE: + mutated, - unmutated, MSI microsatellite instability; $\delta$-H2AX, RAD51, DNA-pk, FANCD2, and PARP-1: + protein expressed (positive $\mathrm{H}$-score), - negative $\mathrm{H}$-score.

DSB Double Strand Breaks, HR Homologous Recombination, NHEJ Non Homologous End-Joining, NER Nucleotide excision repair, $M M R$ Mismatch Repai, DFS Disease Free Survival. $P$ value $* P<$ $0.05 ; * * P<0.01$ and $* * * P<0.001$

towards improved Disease Free Survival (Table 1). Given the amount of redundancy among DNA repair pathways, we sought to investigate whether a combined approach would be more informative. Non-homologous end-joining is an alternative but error-prone repair pathway for double strand break, which may be particularly relevant in the setting of Homologous Recombination deficiency. We conducted a combined analysis of DNA-pk and FANCD2, positive and negative regulators of Non Homologous End-Joining, respectively. Among DNA-pk + /FANCD2- (Non Homologous End-Joining proficient) tumors, RAD51 loss was associated with significantly improved Disease Free Survival (Hazard Ratio $=0.18 ; p=0.024)$. 


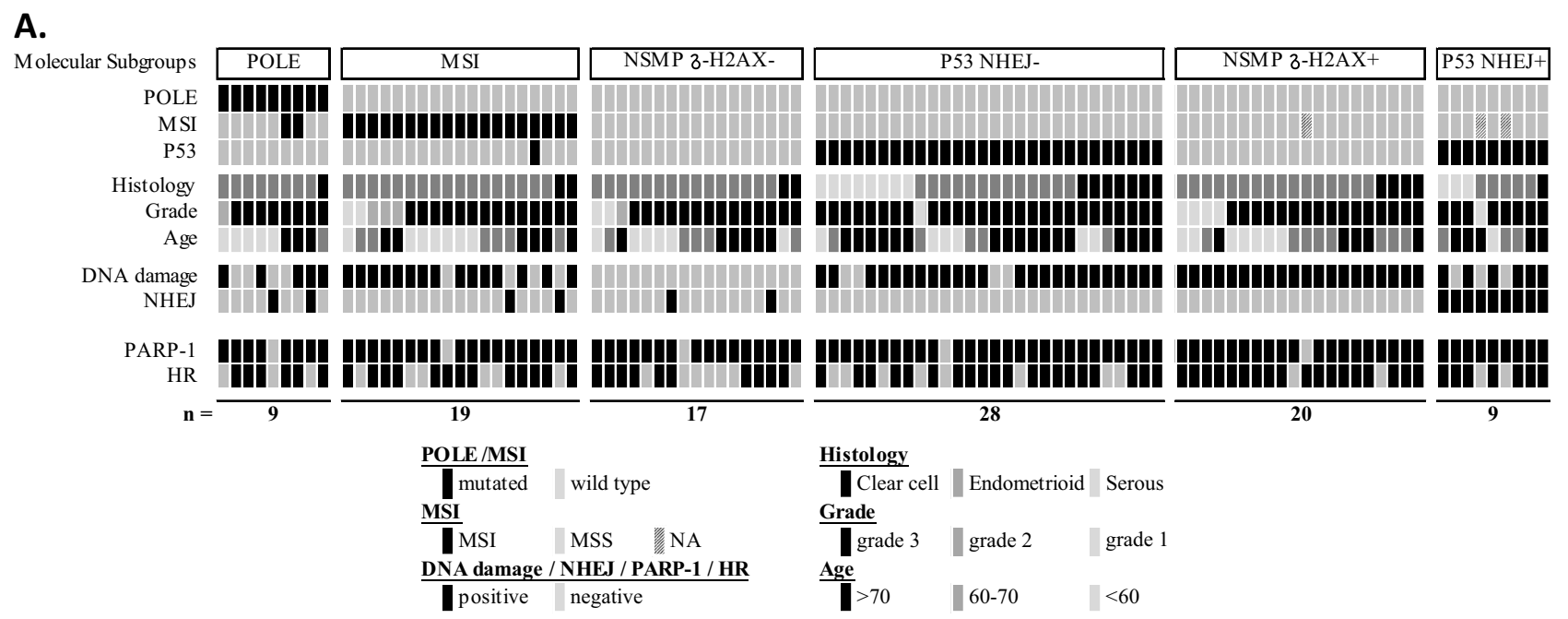

B.

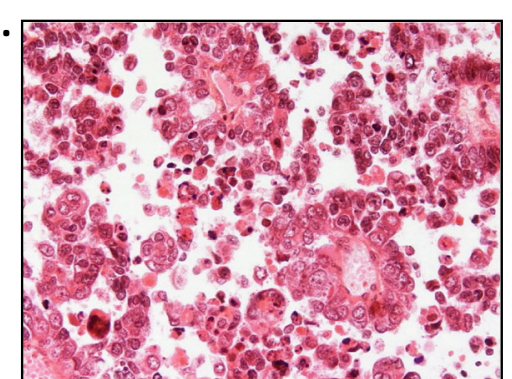

Serous EC

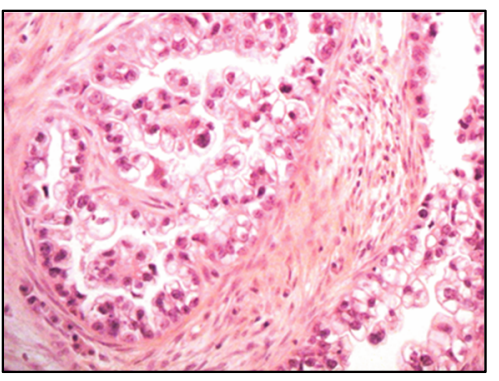

Clear Cells EC

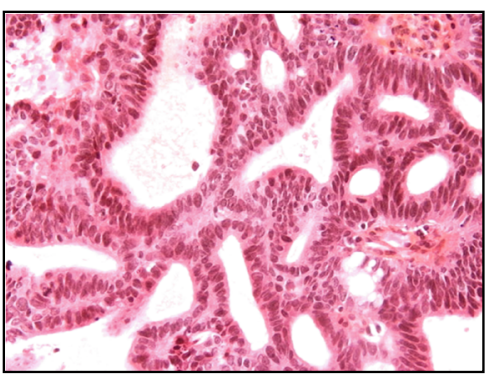

Endometrioid EC
Fig. 2 DNA damage and response landscape of high-risk endometrial cancer. a Immunohistological analysis focusing on DDR biomarkers results in the identification of six molecular subgroups within high-risk endometrial cancer: (1) "POLE mutated" (POLE), (2) "microsatellite instable" (MSI), (3) "no specific molecular profile/Z-H2AX positive" (NSMP -H2AX-), (4) "TP53 mutated/Non Homologous End-Joining negative" (P53 NHEH-), (5) "no specific molecular profile/-H2AX positive" (NSMP -H2AX+), and (6) "TP53 mutated/Non Homologous End-Joining positive" (P53 NHEH+). Tumors FANCD2-/DNA-pk+ were defined as Non Homologous End-Joining positive (NHEJ+); RAD51+ as Homologous Recombination proficient (HR+), - H2AX+ as DNA damage positive. b Representative Hematoxylin Eosin stained picture of the three endometrial carcinomas histotypes included in our cohort: Serous, Clear cells and Endometrioid

\section{Prognostic value of DNA damage and repair biomarkers within molecular subsets of high-risk endometrial cancer}

The "TP53 mutated" subset demonstrated significantly higher levels of DNA damage $(\delta-\mathrm{H} 2 \mathrm{AX})$ and a significantly worse prognosis with a median Disease Free Survival of 36.5 months (Hazard Ratio for Disease Free Survival = 2.06; $p=0.03$ compared to the rest of High-risk endometrial tumors). We therefore sought to determine whether DNA damage response biomarkers could offer further prognostic information above and beyond TP53 alone. Combined TP53 mutation/ $\delta-\mathrm{H} 2 \mathrm{AX}+$ identified a subset $(N$ $=30$ ) with the worst Disease free survival (24.4 months, Hazard Ratio. $=2.7 ; \quad p=0.005)$ and Overall survival (Hazard Ratio $=2.2 ; p=0.021)$ suggesting that the incorporation of a measure of DNA damage could further refine the prognostication of "TP53 mutated" high-risk endometrial cancer (Figure S4.A). RAD51 status did not provide discriminatory prognostic information within the "TP53 mutated" subgroup. However, combining an evaluation of Non Homologous End-Joining-proficiency (DNA-pk +/FANCD2-) with TP53 status had an important impact, with this subgroup showing a drastically worse Disease Free Survival (19.9 months, Hazard Ratio $=4.95 ; p=$ 0.009) demonstrating that incorporation of DNA-pk and FANCD2 with TP53 mutation offered significantly more prognostic information than TP53 mutation alone (Figure S4.A). This resulted in significant refinement of our previously reported classification of high-risk endometrial cancer (Fig. 3).

Classification into "no specific molecular profile" alone was only non-significantly associated with worse Disease Free Survival (31.8 months, Hazard Ratio $=1.7 ; p=0.11$ ). These tumors tended to have lower levels of $\delta-\mathrm{H} 2 \mathrm{AX}$ than TP53m or the "Microsatellite unstable/POLE mutated" subset. However, those "no specific molecular profile" tumors showing detectable DNA damage at diagnosis (no 
A.

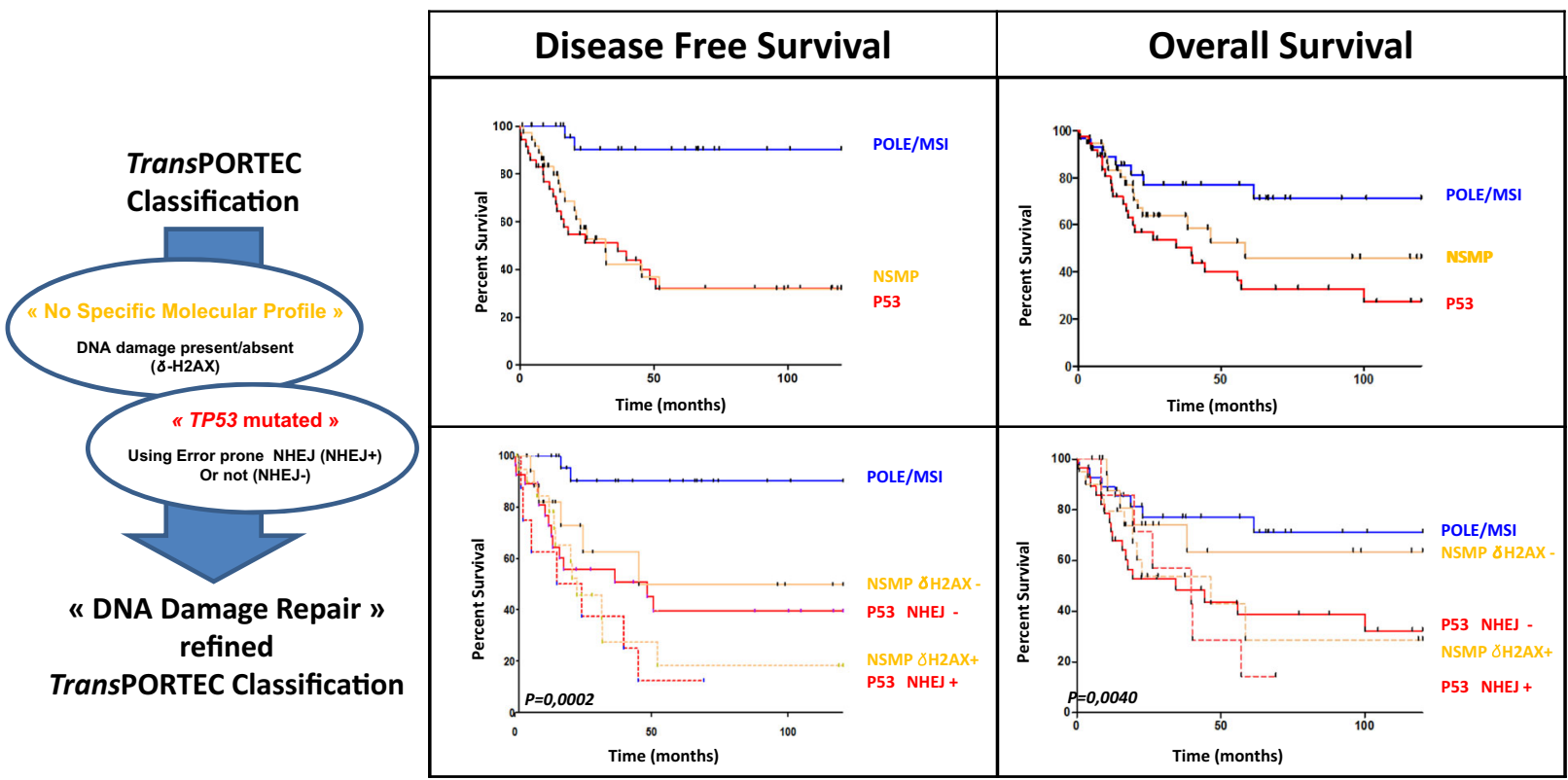

B.

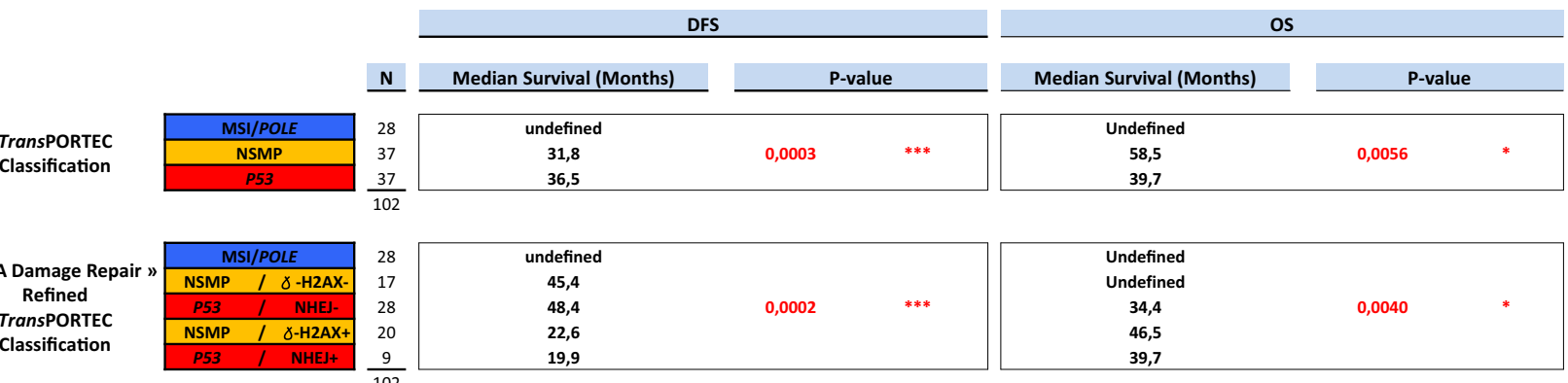

Fig. 3 Poor prognosis endometrial cancer molecular subclasses refinement using DNA Damage and Response bio-markers. Combining an evaluation of Non Homologous End-Joining proficiency (DNA$\mathrm{pk}+/$ FANCD2-) with "TP53 mutated" status and $\delta$-H2AX with "no specific molecular profile" subgroup significantly refined the high-risk endometrial cancer into distinct prognostic subgroups in disease free survival and overall Survival (a graphs and b statistical data).

specific molecular profile/ $\delta-\mathrm{H} 2 \mathrm{AX}+$ ) had significantly shorter Disease Free Survival (22.6 months, Hazard Ratio = $2.56 ; p=0.026$ ) (Figure S4.B). Incorporating an evaluation of $\delta$-H2AX significantly refined the high-risk endometrial cancer into distinct prognostic subgroups (Fig. 3).

Taken together DNA-pk, FANCD2, and $\delta$-H2AX resulted in a new refined classification of the two poor prognostic subsets of high-risk endometrial cancer resulting in five subgroups with significantly different outcomes in terms of Disease free survival (Mantel-Cox test $p=0.0002$ ) (Fig. 3a).

Practically speaking, this new classification might provide useful prognostic information for individual patients. For example, in the case of a "no specific molecular profile" patient, $\delta-\mathrm{H} 2 \mathrm{AX}$ would allow us to determine whether she is in the good or poor prognosis subgroup: if she is $\delta-\mathrm{H} 2 \mathrm{AX}-$, then her Hazard Ratio for disease free survival is 2.01
$\delta$-H2AX + Tumors with DNA damage; $\delta$-H2AX - Tumors without DNA damage, NHEJ+ tumors error prone Non Homologous EndJoining proficient, NHEJ - tumors error prone Non Homologous EndJoining not proficient, MSI microsatellite instability, POLE Polymerase Epsilon mutated, P53 TP53 mutated, DF Sease Free Survival, OS Overall Survival. $P$ value $* P<0.05 ; * * P<0.01$ and $* * * P<0.001$

compared to a "no specific molecular profile" $\delta-\mathrm{H} 2 \mathrm{AX}+$, this information could be incorporated into risk stratification and clinical decision making regarding the need for adjuvant therapy.

Importantly, the inclusion of DNA damage response biomarkers also significantly refined the prognostic classification when using Overall Survival as an endpoint (Fig. 3).

\section{Actionable alterations and immune infiltration within DNA Damage Repair biomarker-refined High- Risk Endometrial Cancer molecular subgroups}

Targeted sequencing using Next Generation Sequencing and immunohistochemistry have been previously conducted on this cohort focusing on oncogenic pathways for which inhibitors are currently available (Table S4). The "no 
specific molecular profile" $\delta$-H2AX negative subgroup showed the greatest enrichment for PI3K pathway alterations $(50 \%)$ and combined estrogen and progesterone receptor $(\mathrm{ER}+/ \mathrm{PR}+)$ positivity $(71 \%)$ suggesting these tumors may benefit from the association of mTOR or AKT inhibitors with endocrine therapies. The "no specific molecular profile" $\delta$-H2AX positive High Risk-Endometrial Cancer had lower levels of actionable PI3K alterations and $\mathrm{ER}+/ \mathrm{PR}+$ positivity, however both "no specific molecular profile" $\delta$-H2AX-positive and -negative tumors demonstrated frequent ARIDIA mutations (25 and 31\%, respectively). ARID1A is implicated in ATM/ATR initiated DNA repair and ARIDIA loss of function mutations have been associated with increased reliance on ATR mediated checkpoint regulation with resulting increased sensitivity to both ATR and PARP inhibitors $[8,9]$.

The "TP53 mutated" Non Homologous End-joining positive subgroup lacked actionable mutations and only $22 \%$ showed combined ER/PR expression. However $\delta$ $\mathrm{H} 2 \mathrm{AX}$ levels were high (median $\mathrm{H}$-score $=55$ ) and all expressed high levels of PARP-1 (H-score range: 87-270) suggesting that these tumors harboring high levels of endogenous DNA damage and favouring the error-prone non homologous end-joining pathway of DNA repair may be particularly responsive to platinum-based chemotherapy or PARP inhibitors.

In addition, we compared T-cell immune infiltrates across our refined high-risk endometrial cancer molecular subgroups (Fig. 4a). As previously published "Microsatellite unstable/POLE mutated" tumors had the highest levels of CD8+ immune cells and PD-1 expression. However, within the "no specific molecular profile" subset, $\delta$ H2AX tumors had significantly higher CD8+ cells (median: 82 vs. 44 months; $p=0.017$, vs. the "no specific molecular profile" $\delta-\mathrm{H} 2 \mathrm{AX}+$ ) suggesting that these may benefit from immune therapies alone or in combination with targeted therapies. The "TP53 mutated" Non Homologous End-Joining negative subgroup showed high levels of CD8 + mmune cell infiltration and higher PD-1 expression than the "no specific molecular profile" and "TP53 mutated" Non Homologous End-Joining positive subsets (Fig. 4). In fact, the level of CD8 and PD-1 expression in "TP53 mutated" Non Homologous End-Joining negative tumors was comparable to the "Microsatellite unstable/POLE mutated" subgroup, providing a rationale for PD-1/PD-L1 inhibitors in this group.

\section{Discussion}

The TransPORTEC consortium was established to refine the prognostic classification and identify novel therapeutic strategies in High-Risk Endometrial Cancer. The TCGA has
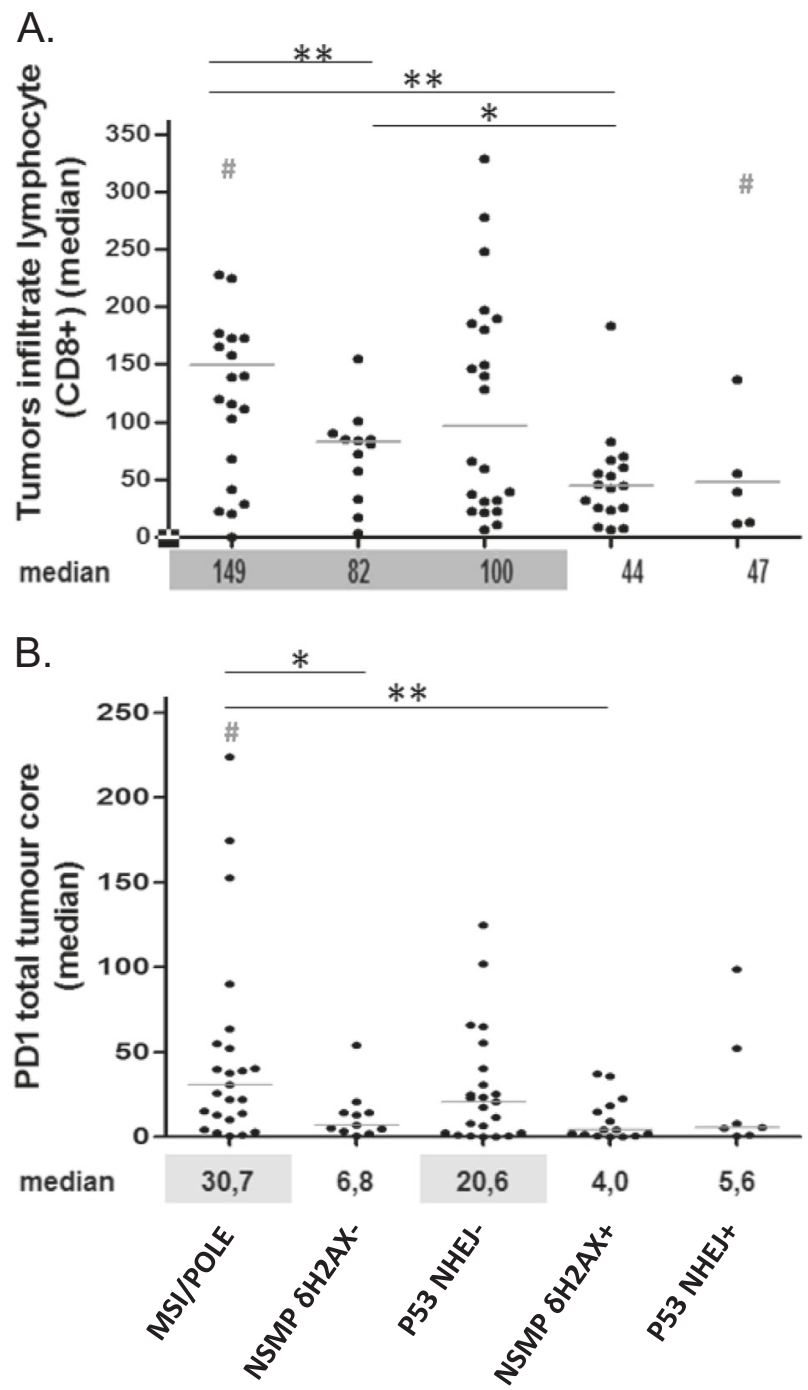

Fig. 4 T-cell immune infiltrates $(\mathrm{CD} 8+)(\mathbf{a})$ and PD-1 expression (b) across our refined high-risk endometrial cancer five molecular subgroups: (1) POLE mutated (POLE), (2) microsatellite instable (MSI), (3) no specific molecular profile/-H2AX positive (NSMP -H2AX-), (4) TP53 mutated/Non Homologous End-Joining negative (P53 NHEJ-), (5) no specific molecular profile/-H2AX positive (NSMP -H2AX+), and (6) TP53 mutated/Non Homologous End-Joining positive (P53 NHEJ+). \#: Out of range data were excluded for graphical representation $\left(\mathrm{N}_{\mathrm{CD} 8}=7\right.$, $\mathrm{N}_{\mathrm{PD}-1}=1$ tumors), but were included for statistical analysis (Mann Whitney test: $p$-value $* p<0.05 ; * * p<0.01$ )

generated important advances into the genomic characterization of Endometrial Cancer, however these studies were conducted in unselected endometrial Cancers, mainly low risk tumors $(69 \%$ stage I and 53\% G1/2 endometrioid histology). The current TransPORTEC High-Risk Endometrial Cancer cohort included $45 \%$ stages III/IV and mainly highrisk histologies (85\% Grade 3 endometrioid, serous or clear cells). We have previously described a clinically applicable molecular classification for endometrial cancers and confirmed the important survival advantage for the "Microsatellite unstable/POLE mutated" molecular subsets in high- 
risk endometrial cancer. In the current study, the "Microsatellite unstable/POLE mutated" subsets demonstrate overlapping Disease free survival curves, whereas in the TCGA cohort, the "POLE mutated" displayed a better prognosis than the "Microsatellite unstable." Most of the patients in the TCGA dataset did not receive adjuvant treatment. Eightynine percent (82/92) of Endometrial Cancer included in the TransPORTEC series received postoperative treatment. The outcome of the "Microsatellite unstable" subset may have been improved by adjuvant strategies, thus converting an aggressive Microsatellite unstable tumor into a clinically favorable one [10]. However, within high-risk endometrial cancer and in contrast to "Microsatellite unstable" and "POLE mutated" subgroups, the "TP53 mutated" and "no specific molecular profile" subgroups had remarkably similar poor disease free survival (median disease free survival $=36$ and 32 months, respectively), which may be attributed to the much higher proportion of non endometrioid histologies (Clear Cells and serous) compared to the TCGA cohort. DNA repair defects via Mismatch Repair have been clearly implicated in both $P O L E$ and MSI endometrial tumors and result in high mutation loads. TP53 mutated endometrial tumor is typically genomically unstable which could be attributed to impaired double strand DNA breaks repair. The first aim of the current study was to investigate whether biomarkers implicated in double strand break detection and repair could further refine the prognostic classification of the "no specific molecular profile" and "TP53 mutated" molecular subsets.

We have shown for the first time that incorporation of DNA damage response biomarkers results in significant refinement of the TransPORTEC molecular classification of high-risk endometrial cancer using affordable and clinically feasible biomarkers. This new DNA damage responserefined molecular classification produced 5 distinct DNA damage response driven prognostic subgroups with worsening survival: group1 "Microsatellite unstable/POLE mutated"; group2 "no specific molecular profile/ $\delta$-H2AX negative"; group3 "TP53 mutated/Non Homologous EndJoining negative"; group4 "no specific molecular profile/ $\delta$ H2AX positive"; and group5 "TP53 mutated/Non Homologous End-Joining positive" ( $p=0.0002)$.

In this study we analyzed double strand breaks DNA damage $(\delta-\mathrm{H} 2 \mathrm{AX})[11,12]$ and repair (RAD51, DNA-pk and FANCD2) biomarkers, as well as PARP-1 as a target for PARP inhibitors (PARPi). TP53 mutated high-risk endometrial cancer showed significantly higher levels of $\delta$ $\mathrm{H} 2 \mathrm{AX}$, providing evidence of its value as a marker of DNA damage.

DNA damage response pathways protect the genome by repairing lesions, such as double strand breaks which are common events caused by endogenous and exogenous DNA damage. Here, we demonstrated for the first time that at diagnosis a significant proportion of high-risk endometrial cancer were deficient for DNA Damage Response biomarkers. RAD51 recombinase is the key downstream effector of the homologous recombination pathway of double strand breaks repair, and $27 \%$ of high-risk endometrial cancer demonstrated complete loss of nuclear RAD51, suggesting possible Homologous Recombination deficiency. In contrast fewer than 5\% showed loss of the target for PARP inhibitors, nuclear PARP-1. Forty percent showed lack of DNA-dependent protein kinase (DNA-pk) [13], an important regulator of the cellular response to double strand DNA breaks that promotes Non Homologous End-Joining. A similar number of high-risk-endometrial cancer demonstrated loss of FANCD2, a suppressor of inappropriate DNA repair by the Non Homologous EndJoining machinery $[14,15]$. Thus, combined DNA-pkpositivity with FANCD2 loss was used as surrogate marker for error prone Non Homologous End-Joining.

TP53 mutation is a powerful prognostic marker both in unselected, mainly low risk Endometrial Cancer (TCGA) and remains significant among high-risk endometrial cancer. However, not all TP53 mutations are equal, as supported by the significant number of TP53 mutations described among good prognosis "POLE mutated" endometrial tumors $[16,17]$. Including markers of Non Homologous End-Joining (DNA-pk+/FANCD2-) significantly added to the prognostic information provided by TP53 mutation alone segregating the "TP53 mutated" tumors into two classes with widely divergent outcomes (20 months vs. 48 months-Fig. 3b)

Similarly, the "no specific molecular profile" group taken as a whole had an equivalent prognosis to "TP53 mutated," however among those "no specific molecular profile" tumors exhibiting detectable endogenous DNA damage, Disease free survival was significantly worse (23 months vs. 45 months-Fig. 3b). The inclusion of DNA damage response biomarkers allowed a refined prognostic classification that predicted disease free survival as well as overall survival (Fig. 3).

We have previously shown that the two good prognostic subgroups, "Microsatellite unstable/POLE mutated" show significantly higher immune infiltration and PD-1 and PD-L1 expression and could benefit from immune targeted strategies. Here the second objective was to further characterize the "no specific molecular profile" and "TP53 mutated" subsets in an effort to determine which of these poor prognosis tumors could be selected for chemotherapy, immune therapies or other targeted agents.

Firstly, our findings generate the hypothesis that DNA damage response biomarkers may be informative to select patients for chemotherapy or DNA damaging agents such as PARP inhibitors. Functional RAD51 foci formation assays 
have been validated as a surrogate functional read-out for Homologous Recombination competency. Lack of RAD51 foci formation has been shown to correlate with high grade, chemotherapy sensitivity and PARP inhibitor sensitivity [18, 19]. While RAD51 is usually evaluated post DNA damaging treatment, the fact that most RAD51 null tumors in our study also showed detectable endogenous DNA damage $(\delta-\mathrm{H} 2 \mathrm{AX}+)$ suggests that they were unable to recruit HR in the face of Double-Strand DNA Breaks. In lung cancer, RAD51 loss has been associated with poor prognosis in untreated tumors, but predictive of benefit from adjuvant platinum-based chemotherapy or radiotherapy [20, 21]. Whether RAD51 loss by immunohistochemistry might identify high-risk endometrial cancer most likely to benefit from platinum-based chemotherapy or PARP inhibitors merits further investigation.

In addition, it is likely that a comprehensive evaluation of DNA repair competency may be more discriminatory. For example, functional Non Homologous End-Joining has been identified as a requirement for PARP inhibitor sensitivity, and Non Homologous End-Joining defective cancers are resistant to PARP inhibitors, even in the setting of BRCA mutation [22-24]. PARP-1 overexpression has been associated with poor prognosis, resistance to platinum chemotherapy but increased responsiveness to PARP inhibitors $[25,26]$. As such the "TP53 mutated"/Non Homologous End-Joining negative subset are likely to be resistant to PARP inhibitors, while the combined RAD51-/Non Homologous End-Joining+/PARP-1+ tumors may be the most responsive.

Intriguingly we have previously reported that despite low neoantigen loads, a subset of "no specific molecular profile" and "TP53 mutated" high-risk endometrial tumors displayed high CD8+ immune infiltrates including PD-1+ immune cells. Within the "no specific molecular profile" subset, lack of endogenous double-strand breaks DNA damage $(\delta$ H2AX-negative) was significantly associated with higher $\mathrm{CD} 8+\mathrm{T}$ cells and improved prognosis (compared to "no

\begin{tabular}{|c|c|c|c|c|c|}
\hline A. & Good Prognosis & & . & $\longrightarrow \mathrm{P}$ & rognosis \\
\hline $\begin{array}{r}\text { DDR defined } \\
\text { molecular } \\
\text { Subgroups }\end{array}$ & POLE or MSI & $\begin{array}{l}\text { NSMP } \\
\delta-H 2 A X-\end{array}$ & $\begin{array}{l}\text { P53 } \\
\text { NHEJ- }\end{array}$ & $\begin{array}{l}\text { NSMP } \\
\delta-H 2 A X+\end{array}$ & $\begin{array}{l}\text { P53 } \\
\text { NHEJ+ }\end{array}$ \\
\hline$N=102$ & $27 \%$ & $17 \%$ & $27 \%$ & $20 \%$ & $9 \%$ \\
\hline $\begin{array}{l}\text { Targetable } \\
\text { alterations }\end{array}$ & & $\begin{array}{c}\text { ER+/PR+ } \\
\text { PI3K/AKT pathway }\end{array}$ & $\begin{array}{c}\text { ER+/PR+ } \\
\text { PI3K/AKT pathway }\end{array}$ & $\begin{array}{c}\text { ARID1A \& } \\
\text { PTEN alterations }\end{array}$ & $\begin{array}{c}100 \% \\
\text { PARP-1+ } \\
\text { / NHEJ+ }\end{array}$ \\
\hline CD8 + & high & high & high & & \\
\hline PD-1 + & high & & high & & \\
\hline $\begin{array}{r}\text { Therapeutical } \\
\text { strategies }\end{array}$ & Anti PD-1 / PD-L1 & $\&$ & $\begin{array}{l}\text { herapy } \\
\text { e Therapy }\end{array}$ & $\begin{array}{l}\text { ATRi } \\
\text { PARPi }\end{array}$ & $\begin{array}{l}\text { PARPi } \\
\& \\
\text { Platinum }\end{array}$ \\
\hline
\end{tabular}

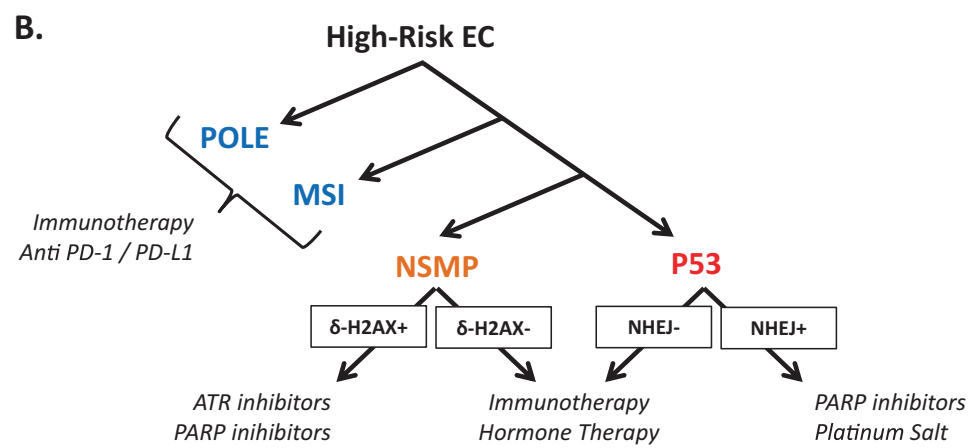

Fig. 5 Refined classification of high-risk endometrial cancer based on DNA damage response biomarkers with specific therapeutic strategies associated. High-risk endometrial cancer molecular subgroups characteristics (a) and algorithm (b) using DNA damage response biomarkers. "No specific molecular profile" and "TP53 mutated" molecular subgroups were refined according to their DNA damage response positivity/negativity using respectively "DNA damage" and "error prone Non Homologous End-Joining" biomarkers: (1) POLE mutated (POLE)/microsatellite instable (MSI), (2) no specific mole-

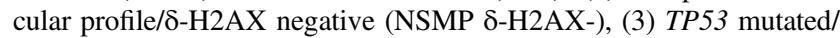
Non Homologous End-Joining negative (P53 NHEJ-), (4) no specific molecular profile/ $\delta$ - $\mathrm{H} 2 \mathrm{AX}$ positive (NSMP $\delta-\mathrm{H} 2 \mathrm{AX}+$ ), and (5) TP53 mutated/Non Homologous End-Joining positive (P53 NHEJ+). «Error-prone Non Homologous End-Joining» positive defined as "DNA-pk+/FANCD2-»; «DNA damaged» defined as $\delta$-H2AX positive 
specific molecular profile"/ $\delta-\mathrm{H} 2 \mathrm{AX}+$ ). Among "TP53 mutated" tumors, Non Homologous End-Joining negative tumors showed dense CD8 infiltration, markedly elevated PD-1 expression and significantly improved prognosis compared to "TP53 mutated"/Non Homologous End-Joining positive. These data suggest that DNA damage responserefined classification of high-risk endometrial cancer can provide prognostic information as well as define molecular subsets beyond "Microsatellite unstable/POLE mutated" tumors who could benefit from immune strategies.

In conclusion, we present a refined classification of highrisk endometrial cancer based on DNA damage response biomarkers which offers both prognostic as well as molecular subtype specific therapeutic strategies. In addition to the well described "Microsatellite unstable/POLE mutated" subsets who may benefit from PD-1/PD-L1 inhibitors, Fig. 5 illustrates how one could envision including DNA damage response biomarkers to orient high-risk Endometrial Cancer patients toward selected therapeutic strategies. These are merely speculative but could be tested in future molecularly guided trials:

The intermediate prognosis lymphocyte-rich "No Specific Molecular Profile/ $\delta$-H2AX negative" tumors may be oriented toward hormonal or immune-oncology treatments,

The intermediate prognosis PD1-high "TP53 mutated/ Non Homologous End-Joining negative" likely to be resistant to PARP inhibitors may be offered PD-L1/PD-1 inhibitors,

The poor prognosis lymphocyte poor "No Specific Molecular Profile/ $\delta$-H2AX positive" showing high levels of endogenous DNA damage and frequent ARID1A or PTEN alteration may be suited to ATR or PARP inhibitors, and

The worst prognosis lymphocyte-poor "TP53 mutated/ Non Homologous End-Joining positive" harboring high levels of endogenous DNA damage, favouring the errorprone Non Homologous End-Joining pathway of DNA repair may be responsive to platinums or PARP inhibitors.

As part of a continued collaboration within the TransPORTEC consortium, a comprehensive evaluation of proteomic and genomic DNA Damage Repair biomarkers will be conducted on high-risk endometrial cancer tumor samples from the recently completed randomized PORTEC3 trial in order to identify predictors of poor prognosis but preferential benefit from adjuvant chemotherapy and identify candidate biomarkers for PARP inhibitor sensitivity. These will then require prospective validation in the ongoing and planned trials of PARP inhibitors in advanced Endometrial Cancer.
Acknowledgements We acknowledge all clinicians, technicians, scientists, and pathologists involved in the collaborating centers within the participating group in the TransPORTEC consortium.

\section{Compliance with ethical standards}

Conflict of interest The authors declare that they have no conflict of interest.

\section{References}

1. Aalders J, Abeler V, Kolstad P, et al. Postoperative external irradiation and prognostic parameters in stage I endometrial carcinoma: clinical and histopathologic study of 540 patients. Obstet Gynecol. 1980;56:419-27.

2. ASTEC/EN.5 Study Group, Blake P, Swart AM, et al. Adjuvant external beam radiotherapy in the treatment of endometrial cancer (MRC ASTEC and NCIC CTG EN.5 randomised trials): pooled trial results, systematic review, and meta-analysis. Lancet. 2009;373:137-46.

3. De Boer S, Powell ME, Mileshkin L, et al. Adjuvant chemoradiotherapy versus radiotherapy alone for women with high-risk endometrial cancer (PORTEC-3): final results of an international, open-label, multicentre, randomised, phase 3 trial. Lancet Oncol. 2018;19:295-309.

4. Cancer Genome Atlas Research Network, Kandoth C, Schultz N, et al. Integrated genomic characterization of endometrial carcinoma. Nature. 2013;497:67-73.

5. Talhouk A, McAlpine JN. New classification of endometrial cancers: the development and potential applications of genomicbased classification in research and clinical care. Gynecol Oncol Res Pract. 2016;3:14.

6. Stelloo E, Bosse T, Nout RA, et al. Refining prognosis and identifying targetable pathways for high-risk endometrial cancer; a TransPORTEC initiative. Mod Pathol. 2015;28:836-44.

7. Eggink FA, Van Gool IC, Leary A, et al. Immunological profiling of molecularly classified high-risk endometrial cancers identifies POLE-mutant and microsatellite unstable carcinomas as candidates for checkpoint inhibition. Oncoimmunology. 2017;6:e1264565.

8. Lord CJ, Tutt ANJ, Ashworth A. Synthetic lethality and cancer therapy: lessons learned from the development of PARP inhibitors. Annu Rev Med. 2015;66:455-70.

9. Shen J, Peng Y, Wei L, et al. ARID1A deficiency impairs the DNA damage checkpoint and sensitizes cells to PARP inhibitors. Cancer Discov. 2015;5:752-67.

10. McMeekin DS, Tritchler DL, Cohn DE, et al. Clinicopathologic significance of mismatch repair defects in endometrial cancer: an NRG oncology/gynecologic oncology group study. J Clin Oncol. 2016;34:3062-8.

11. Kuo LJ, Yang L-X. Gamma-H2AX - a novel biomarker for DNA double-strand breaks. Vivo. 2008;22:305-9.

12. Siddiqui MS, François M, Fenech MF, et al. Persistent $\gamma \mathrm{H} 2 \mathrm{AX}$ : a promising molecular marker of DNA damage and aging. Mutat Res Rev Mutat Res. 2015;766:1-19.

13. Lieber MR. The mechanism of double-strand DNA break repair by the nonhomologous DNA end-joining pathway. Annu Rev Biochem. 2010;79:181-211.

14. Adamo A, Collis SJ, Adelman CA, et al. Preventing nonhomologous end joining suppresses DNA repair defects of Fanconi anemia. Mol Cell. 2010;39:25-35.

15. Pace P, Mosedale G, Hodskinson MR, et al. Ku70 corrupts DNA repair in the absence of the Fanconi anemia pathway. Science. 2010;329:219-23. 
16. Morice P, Leary A, Creutzberg C, et al. Endometrial cancer. Lancet. 2016;387:1094-108.

17. Schultheis AM, Martelotto LG, De Filippo MR, et al. TP53 mutational spectrum in endometrioid and serous endometrial cancers. Int J Gynecol Pathol. 2016;35:289-300.

18. Mukhopadhyay A, Elattar A, Cerbinskaite A, et al. Development of a functional assay for homologous recombination status in primary cultures of epithelial ovarian tumor and correlation with sensitivity to poly(ADP-ribose) polymerase inhibitors. Clin Cancer Res. 2010;16:2344-51.

19. Graeser M, McCarthy A, Lord CJ, et al. A marker of homologous recombination predicts pathologic complete response to neoadjuvant chemotherapy in primary breast cancer. Clin Cancer Res. 2010;16:6159-68.

20. Gachechiladze M, Škarda J, Soltermann A, et al. RAD51 as a potential surrogate marker for DNA repair capacity in solid malignancies. Int J Cancer. 2017;141:1286-94.

21. Gachechiladze M, Škarda J, Kolek V, et al. Prognostic and predictive value of loss of nuclear RAD51 immunoreactivity in resected non-small cell lung cancer patients. Lung Cancer Amst Neth. 2017;105:31-38.

22. Bouwman P, Aly A, Escandell JM, et al. 53BP1 loss rescues BRCA1 deficiency and is associated with triple-negative and BRCA-mutated breast cancers. Nat Struct Mol Biol. 2010;17:688-95.

23. Yang Z-M, Liao X-M, Chen Y, et al. Combining 53BP1 with BRCA1 as a biomarker to predict the sensitivity of poly(ADPribose) polymerase (PARP) inhibitors. Acta Pharmacol Sin. 2017;38:1038-47.

24. McCormick A, Donoghue P, Dixon M, et al. Ovarian cancers harbor defects in nonhomologous end joining resulting in resistance to rucaparib. Clin Cancer Res. 2017;23:2050-60.

25. Rojo F,García-Parra J,Zazo S, et al. Nuclear PARP-1 protein overexpression is associated with poor overall survival in early breast cancer. Ann Oncol. 2012;23:1156-64.

26. Olaussen KA, Adam J, Vanhecke E, et al. PARP1 impact on DNA repair of platinum adducts: preclinical and clinical read-outs. Lung Cancer Amst Neth. 2013;80:216-22.

\section{Affiliations}

\section{Aurélie Auguste $\mathbb{1}^{1} \cdot$ Catherine Genestie ${ }^{1,2} \cdot$ Marco De Bruyn $^{3} \cdot$ Julien Adam $^{2} \cdot$ Audrey Le Formal $^{1}$.

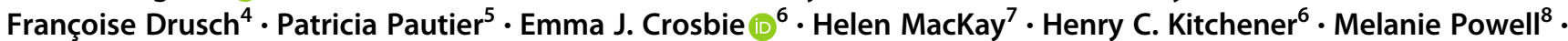 Pamela M. Pollock ${ }^{9} \cdot$ Linda Mileshkin $^{10} \cdot$ Richard J. Edmondson $^{10,11} \cdot$ Remi Nout $^{12} \cdot$ Hans W. Nijman $^{13}$. Carien L. Creutzberg ${ }^{12} \cdot$ Tjalling Bosse $^{14} \cdot$ Alexandra Leary $^{1,5}$}

1 Gustave Roussy, Gynecology Unit, U981 INSERM, Villejuif, France

2 Gustave Roussy, Department of Medical Oncology, Pathology department, Villejuif, France

3 Department of Obstetrics \& Gynecology, University Medical Center Groningen, University of Groningen, Groningen, The Netherlands

4 Gustave Roussy, Histo-Cyto Pathology platform, Villejuif, France

5 Department of Medical Oncology, Gustave Roussy, Villejuif, France

6 Institute of Cancer Sciences, University of Manchester, St Mary's Hospital, Manchester, UK

7 Department of Obstetrics and Gynecology, Princess Margaret Hospital/University Health Network, University of Toronto, Division of Gynecologic Oncology, Toronto, Canada
8 Department of Clinical Oncology, arts Health NHS Trust, London, UK

9 Institute of Health and Biomedical Innovation, Queensland University of Technology, Brisbane, Australia

10 Peter Mac Callum Cancer Centre, Division of Cancer Medicine, East Melbourne, Australia

11 Institute of Cancer Sciences, University of Manchester, St Marys Hospital, Manchester, UK

12 Department of Clinical Oncology, eiden University Medical Center, Leiden, The Netherlands

13 Department of Gynecology, University Medical Center Groningen, University of Groningen, Groningen, The Netherlands

14 Department of Pathology, Leiden University Medical Center, Leiden, The Netherlands 